\title{
Choosing New Therapies for Gonorrhoea: We Need to Consider the Impact on the Pan-Neisseria Genome. A Viewpoint
}

\author{
Chris Kenyon ${ }^{1,2,3, *(\mathbb{D})}$, Jolein Laumen ${ }^{1}$ and Sheeba Manoharan-Basil ${ }^{1}$ (D) \\ 1 HIV/STI Unit, Department of Clinical Sciences, Institute of Tropical Medicine, 2000 Antwerp, Belgium; \\ jlaumen@itg.be (J.L.); sbasil@itgg.e (S.M.-B.) \\ 2 Division of Infectious Diseases and HIV Medicine, University of Cape Town, Anzio Road, Observatory, \\ Cape Town 7701, South Africa \\ 3 STI Reference Center, Department of Clinical Sciences, Institute of Tropical Medicine, 2000 Antwerp, Belgium \\ * Correspondence: ckenyon@itg.be; Tel.: +32-3-248-0796; Fax: +32-3-248-0831
}

Citation: Kenyon, C.; Laumen, J.; Manoharan-Basil, S. Choosing New Therapies for Gonorrhoea: We Need to Consider the Impact on the Pan-Neisseria Genome. A Viewpoint. Antibiotics 2021, 10, 515. https:// doi.org/10.3390/antibiotics10050515

Academic Editor:

Myron Christodoulides

Received: 26 March 2021

Accepted: 27 April 2021

Published: 1 May 2021

Publisher's Note: MDPI stays neutral with regard to jurisdictional claims in published maps and institutional affiliations.

Copyright: (c) 2021 by the authors. Licensee MDPI, Basel, Switzerland. This article is an open access article distributed under the terms and conditions of the Creative Commons Attribution (CC BY) license (https:// creativecommons.org/licenses/by/ $4.0 /)$.

\begin{abstract}
The development of new gonorrhoea treatment guidelines typically considers the resistanceinducing effect of the treatment only on Neisseria gonorrhoeae. Antimicrobial resistance in N. gonorrhoeae has, however, frequently first emerged in commensal Neisseria species and then been passed on to N. gonorrhoeae via transformation. This creates the rationale for considering the effect of gonococcal therapies on resistance in commensal Neisseria. We illustrate the benefits of this pan-Neisseria strategy by evaluating three contemporary treatment options for N. gonorrhoeae-ceftriaxone plus azithromycin, monotherapy with ceftriaxone and zoliflodacin.
\end{abstract}

Keywords: N. gonorrrhoeae; Neisseria; antimicrobial resistance; antimicrobial consumption; dual therapy

\section{Introduction}

Neisseria gonorrhoeae (NG) causes the disease gonorrhoea, which is an important cause of urethritis, cervicitis and proctitis. NG has developed resistance to every class of antimicrobial used to treat it [1]. Antimicrobial resistance (AMR) is not, however, inevitable. There are large differences in the prevalence of AMR between different countries [2]. In some populations, such as the Northern Territories of Australia, there is so little AMR that oral penicillin can still be used to treat NG [2,3]. In other countries such as China, high levels of resistance to azithromycin (18.6\%) and reduced susceptibility to ceftriaxone (12.2\%) compromise even these last-line, single-dose therapies [4]. In general, the prevalence of AMR to a range of antibiotics has increased in most countries. Thus, the World Health Organization's Global Gonococcal Antimicrobial Surveillance Programme (GASP) has found continuing high-level resistance to penicillin, tetracycline and ciprofloxacin around the world. It has also found increasing resistance to azithromycin and the emergence of decreased susceptibility and resistance to cephalosporins such as cefixime and ceftriaxone [5]. Cephalosporin and macrolide resistance in NG is of particular concern. In the United States (USA), the percentage of isolates with reduced susceptibility (MIC $\geq 2.0 \mu \mathrm{g} / \mathrm{mL}$ ) increased from $0.6 \%$ in 2013 to $4.6 \%$ in 2018 [6]. Likewise, in the 24 countries participating in the European Gonococcal Antimicrobial Surveillance Programme (Euro-GASP), azithromycin resistance increased from 5.3\% in 2011 to $13.3 \%$ in 2018 (MIC > $1.0 \mu \mathrm{g} / \mathrm{mL}$ ) [6]. Whilst there have only been a limited number of isolates with ceftriaxone reported from Europe and the USA, the high prevalence of reduced susceptibility to ceftriaxone reported from China and elsewhere is a major concern [4].

The key determinant of AMR is antimicrobial exposure [7]. As the antimicrobials used to treat gonorrhoea are an important driver of this antimicrobial exposure, treatment guidelines are based in part on minimising the probability that they will induce AMR in NG [6,8]. Treatment of NG will, however, also select for AMR in commensal Neisseria, which are an important component of the oropharyngeal microbiome. These commensal Neisseria 
can then transfer this resistance to NG [9]. Since horizontal gene transfer (HGT) has played a crucial role in the genesis of AMR in NG, we make a case for explicitly incorporating treatment effects on commensal Neisseria when evaluating prospective therapies for NG. We describe this as the pan-Neisseria perspective.

\section{Contemporary Monospecies Approach}

Treatment guidelines for gonorrhoea consider the risk that new treatments could induce AMR but typically only consider this effect on NG. For example, recent United States CDC and European IUSTI NG treatment guidelines both consider resistance induction in their recommended treatment guidelines. Neither includes commensal Neisseria in this evaluation [6,8]. In the case of the CDC guidelines, "concerns regarding potential harm to the microbiome" are listed as one of the reasons for changing recommended treatment from ceftriaxone plus azithromycin (dual therapy) to ceftriaxone (monotherapy) [8]. The IUSTI guidelines focus exclusively on resistance induction in NG and argue that because concurrent resistance to ceftriaxone and azithromycin is extremely rare, dual therapy has likely played a role in decreasing the prevalence of resistance to ceftriaxone and cefixime [6]. The authors further note that there have been examples of therapy failure with monotherapy, but none with dual therapy (Figure 1). These are among the arguments used to recommend dual therapy as the preferred therapy for NG in the IUSTI guidelines. Thus, whilst the CDC explicitly recommends monotherapy, IUSTI recommends dual therapy.

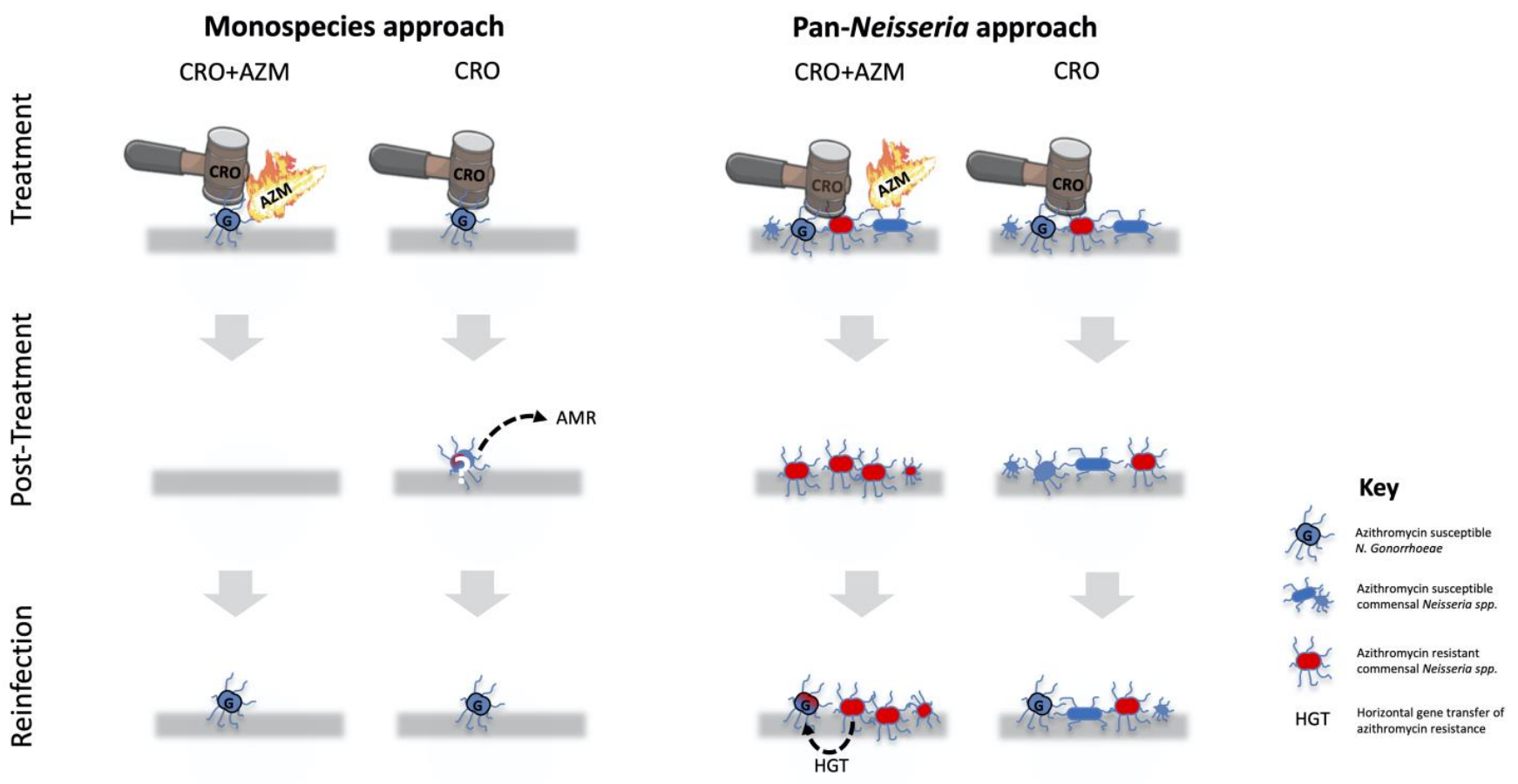

Figure 1. A schematic illustration of select differences between the monospecies and pan-Neisseria approaches to evaluating the risk of inducing resistance of proposed antimicrobial therapy for N. gonorrhoeae (NG/G). The monospecies approach only considers the effects of the treatment on NG. It thus favours dual therapy with ceftriaxone (CRO) and azithromycin (AZM), as this combination minimises the risk of treatment failure that could result in resistance. In the pan-Neisseria approach, monotherapy is favoured, as this is highly efficacious at eradicating NG and does this without selecting for widespread resistance (red bacteria) to macrolides in commensal Neisseria 14 days post-therapy (second panel). The pan-Neisseria, but not the monospecies approach, is cognisant of the risk of the genes conferring macrolide resistance in commensal Neisseria being passed on to a NG reinfection via horizontal gene transfer (HGT; third panel). The rationale for only representing $\mathrm{CRO}$ and $\mathrm{CRO} / \mathrm{AZM}$ as treatment options is that these are the predominant treatments currently recommended by the United States CDC and European IUSTI guidelines. 
It is important to note that approaches that only consider resistance induction in the target organism (mono-species approaches) may be more likely to favour poly-therapy than approaches that consider resistance induction in multiple organisms (Table 1). This is evident if we consider the example of therapy for Mycobacterium tuberculosis, wherein standard, four-drug treatment dramatically reduces the probability of the emergence of AMR compared to single drug therapy [10]. Four drug therapy reduces the probability of AMR emerging. This relates to the fact that a single mycobacterium is considerably less likely to be resistant to four drugs than one drug. Resistance is caused by chromosomal mutations, which occur at a frequency of approximately $10^{-6}$ mycobacterial replications [10]. The probability of a single mycobacterium being resistant to one antimicrobial is thus around $10^{-6}$, but this jumps to $10^{-12}$ if two antimicrobials are considered [11]. This explains why, when single drug therapy with streptomycin was introduced, it was rapidly followed by resistance to streptomycin, which was not the case with quadri-therapy [10]. A key conclusion is that for organisms like M. tuberculosis, where AMR is acquired via chromosomal mutations, monotherapy selects for, and quadri-therapy prevents, the emergence of AMR. The same principles have been shown to apply to other organisms wherein HGT is not prominent such as other species of mycobacterium and Plasmodium falciparum [12]. The situation is, however, more complicated in bacteria like Neisseria spp., wherein HGT plays an important role in the genesis of AMR [13].

Table 1. Select differences between the monospecies and pan-Neisseria approaches to selecting new N. gonorrhoeae (NG) therapies.

\begin{tabular}{ccc}
\hline & Monospecies Approach & Pan-Neisseria Approach \\
\hline Conceptual framework & $\begin{array}{c}\text { Monospecies conception: NG is a } \\
\text { pathogen, and its control requires } \\
\text { optimisation of seek } \\
\text { and destroy activities. }\end{array}$ & $\begin{array}{c}\text { Ecological conception: Commensal Neisseria are } \\
\text { important constituents of a healthy microbiome } \\
\text { and can be a source of AMR for NG and N. } \\
\text { meningitidis. Excessive seek and destroy } \\
\text { activities could induce AMR in commensals, } \\
\text { which could be transferred to NG. }\end{array}$ \\
$\begin{array}{c}\text { Approach to dual therapy } \\
\text { (ceftriaxone + azithromycin) vs. } \\
\text { monotherapy (ceftriaxone) for NG }\end{array}$ & $\begin{array}{c}\text { Treatment with dual therapy is favoured, } \\
\text { as this is more likely to eradicate NG } \\
\text { than monotherapy. }\end{array}$ & $\begin{array}{c}\text { Dual therapy for NG is more likely to have a } \\
\text { negative effect on commensals (composition and } \\
\text { macrolide resistance) and hence, monotherapy } \\
\text { may be preferable. }\end{array}$ \\
\hline AMR Surveillance & $\begin{array}{c}\text { Surveillance in samples of NG is } \\
\text { sufficient, e.g., Euro GASP, } \\
\text { GRASP methodologies }\end{array}$ & $\begin{array}{c}\text { Surveillance should be done in both NG and } \\
\text { commensal Neisseria in core groups, e.g., } \\
\text { culture/MIC of commensal Neisseria from throat } \\
\text { swabs of 30 PrEP clients per centre once a year. }\end{array}$ \\
\hline
\end{tabular}

\section{Pan-Neisseria Approach}

The antimicrobials used to treat NG select for AMR in both pathogenic and commensal Neisseria. This effect has been shown for cephalosporins, fluoroquinolones and macrolides [14-16]. The genetic mutations responsible for this AMR can be readily taken up from these commensals by NG via transformation [17].

This process of transformation has played a critical role in the emergence of NG resistance to both cephalosporins and macrolides [17-19]. Mosaic versions of penA are a crucial determinant of gonococcal cephalosporin resistance [17]. These mosaic penAs emerged via multiple independent acquisitions of sections of the pen $A$ gene from a number of commensal Neisseria, including $N$. cinerea, N. mucosa, N. subflava and N. lactamica $[17,20,21]$. Likewise, transformation from commensal Neisseria has played an important role in the emergence of resistance to azithromycin in NG. As an example, NG has taken up portions of the genes coding for the mtrCDE efflux pump from commensal Neisseria $[18,19]$. This has enabled it to more effectively pump out azithromycin from the intracellular compartment and thereby become less susceptible to azithromycin. 
The available evidence suggests that high levels of antimicrobial consumption are the major determinant of the increase in commensal AMR during the past few decades [14,15,22]. There are a number of different types of antimicrobial consumption that can result in commensal AMR (Figure 2), and it is useful to consider this selection pressure at both the individual and population levels [13]. As illustrated in Figures 1 and 3, dual therapy at the level of an infected individual would very likely eradicate NG but not commensal Neisseria. In part, this is related to differences in resilience.

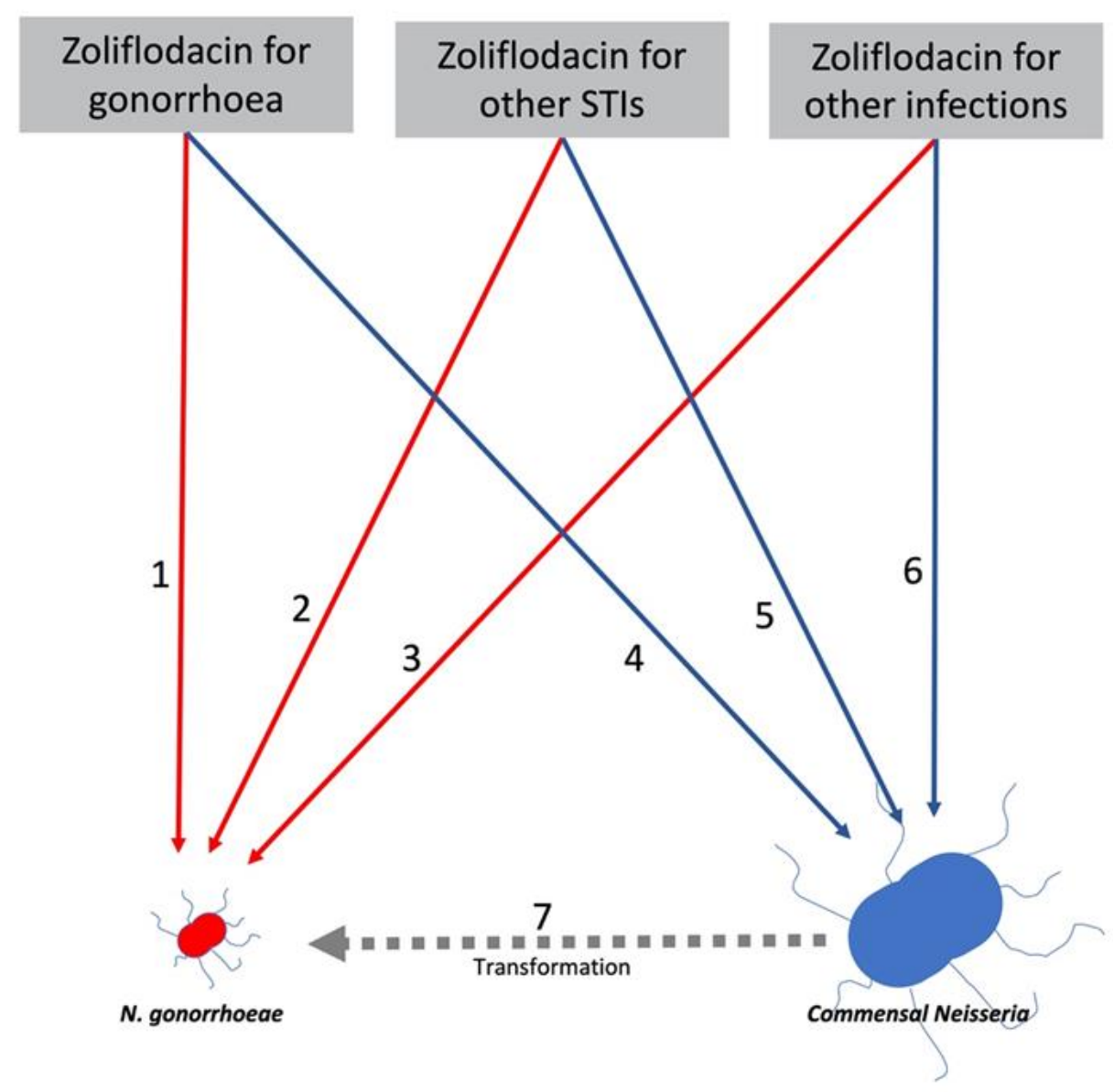

Figure 2. Model of relationship between zoliflodacin consumption for 3 indications and induction of zoliflodacin resistance in N. gonorrhoeae (NG: red) and commensal Neisseria (blue). Zoliflodacin is a promising, novel anti-gonococcal therapy that also shows promise for a range of other infections. Zoliflodacin (ZF) usage could select for AMR directly in NG via 1. ZF used to treat NG, 2. ZF used to treat other STIs and 3. ZF used to treat other infections. It could also select for AMR in commensal Neisseria via 4. ZF used to treat NG, 5. ZF used to treat other STIs or 6. ZF used to treat other infections. Each of these could select for ZF resistance in commensal Neisseria, which could then be transferred to NG via transformation (7). The probability of ZF inducing AMR in NG $(1,2,3)$ and commensal species $(4,5,6)$ could be determined via in vitro experiments. These experiments could also estimate the efficiency of transformation given the co-occurrence of NG and a specific commensal Neisseria (7). The commensal Neisseria in the figure is bigger than NG to reflect the orders of magnitude of the higher prevalence of commensal Neisseria. 


\section{N. gonorrhoeae}
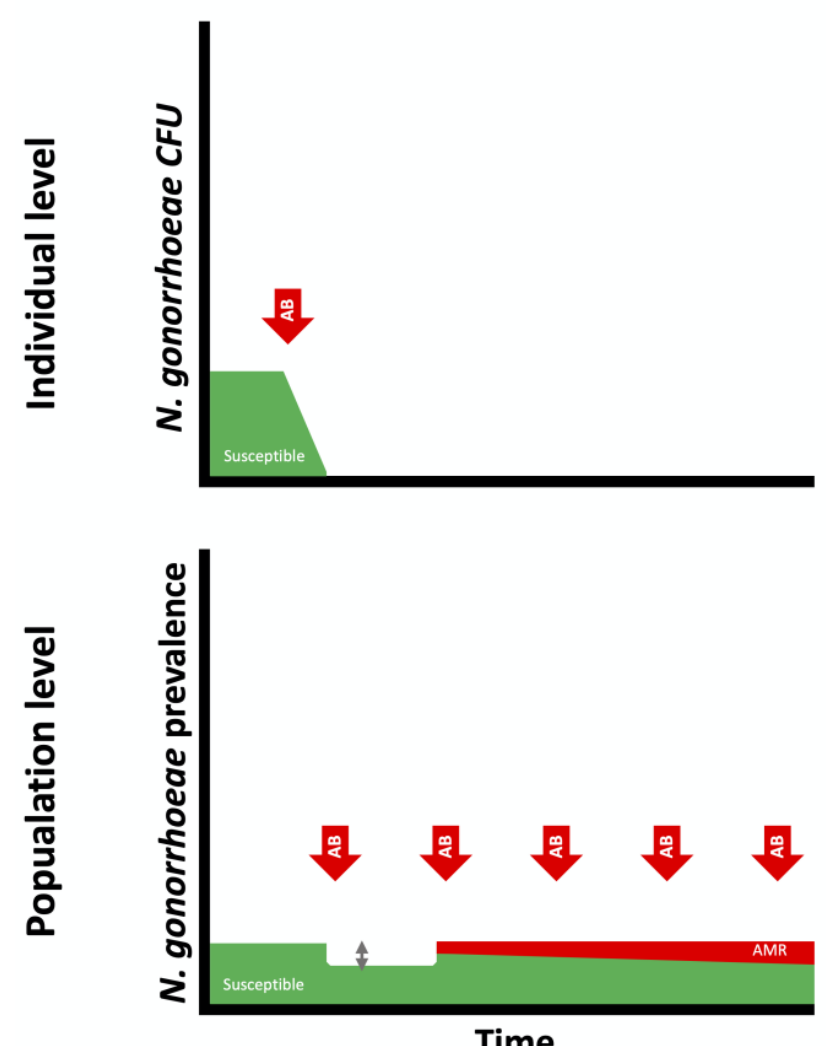

\section{Commensal Neisseria}
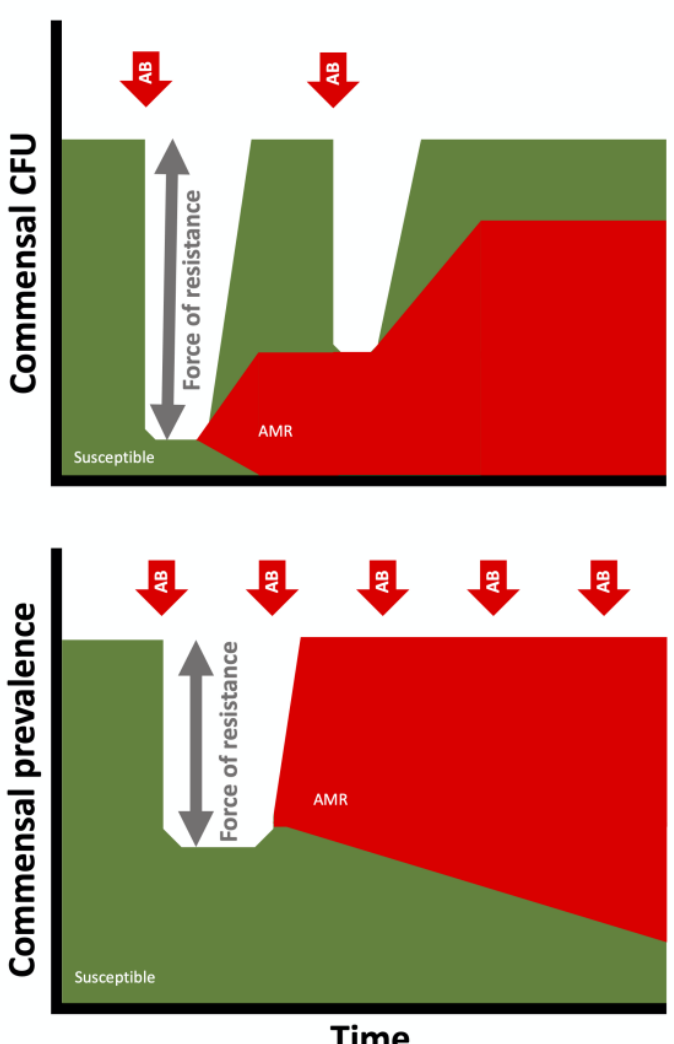

Figure 3. Schematic illustration of the importance of considering the selection of antimicrobial resistance in N. gonorrhoeae (left) and commensal Neisseria (right) at both the individual (top) and population levels (bottom). Whereas dual therapy with ceftriaxone/azithromycin (AB; red arrows) eradicates $N$. gonorrhoeae in an infected individual, it only reduces the abundance of commensal Neisseria. The resilience of commensal Neisseria results in their returning to baseline abundance in a process that selects for resistant isolates. The greater the decline in abundance of commensal abundance, the greater the 'force of resistance' (grey arrows). At a population level, high exposure to ceftriaxone/azithromycin will push the prevalence of both $N$. gonorrhoeae and commensal Neisseria species below the equilibrium prevalences (100\% for commensals and $0.1-10 \%$ for $N$. gonorrhoeae, depending on sexual network connectivity). This will select for resistance to these agents in both $N$. gonorrhoeae and commensal Neisseria. (CFU: colony forming units).

NG at an individual level has little or no resilience. Effective antimicrobial therapy leads to eradication, with close to zero probability of recurrence without reinfection (Figure 4). Commensal Neisseria, in contrast, are highly resilient. Numerous studies have confirmed that close to $100 \%$ of all humans are colonised by specific, commensal Neisseria species in various oropharyngeal niches [23-27]. For example, although their distribution and abundance varied, $N$. mucosa, N. subflava and $N$. flavacens were all found to be present in the oral microbiomes of all persons included in the Human Microbiome Project [28]. This proportion remains remarkably constant despite various antimicrobial challenges [15,25]. For example, the proportion of individuals colonised by commensal Neisseria is unchanged 14 days post-dual ceftriaxone/azithromycin therapy [15,29]. We do not understand the factors underpinning this remarkable resilience of commensal Neisseria, but there is increasing evidence that they are better considered as synergists that provide a number of vital functions to their human hosts [23-27]. Whatever the mechanism, the available evidence suggests that commensal Neisseria are able to withstand broad spectrum antimicrobials and antiseptic mouthwashes that are able to eradicate other bacteria. We hypothesise that the universal presence of commensal Neisseria, combined with their resilience, increases the probability of AMR emerging in high-antimicrobial-exposure settings in two ways. Firstly, the higher prevalence increases the probability of antimicrobial exposure and hence 
bystander AMR selection [30]. This is evident if we consider that a species that is present in $100 \%$ of a population will be much more likely to be exposed to antimicrobials used for any indication than a species whose prevalence is $1 \%$. The second factor is what we have termed the 'force of resistance' effect (Figure 3). This refers to the extent to which antimicrobial consumption depresses a species prevalence below its equilibrium prevalence in a particular population. Consider a species that has an equilibrium prevalence of $100 \%$ and a high propensity to return to $100 \%$ prevalence post-antimicrobial exposure. If antimicrobial therapy drives prevalence down to $20 \%$, this will likely exert a greater selection pressure for the emergence of AMR than if the prevalence was driven down to $95 \%[9,13]$. The 'force of resistance' effect is likely largely determined by the extent to which the prevalence of a bacterial species is reduced below its equilibrium prevalence at a population level or below its normal abundance at an individual level. In the case of NG, the 'force of resistance' only operates at a population level, wherein the equilibrium prevalence of a population is determined by its sexual network connectivity [9]. If this prevalence is reduced with widespread antimicrobial consumption, then the greater the decline, the greater the selection pressure for AMR to emerge (which would enable the NG to return to its equilibrium prevalence; Figure 3 and [9]). In the case of commensal Neisseria, this 'force of resistance' operates at both individual and population levels (Figure 3). One of the population-level mechanisms for AMR to spread within commensals is illustrated in Figure 4, where high antimicrobial consumption leads to relative extinction of susceptible strains and hence an increase in the transmission of resistant strains between individuals via activities such as kissing, which has been shown to be a mechanism for the spread of commensal Neisseria [31,32].

A) Individual Level Selection of AMR

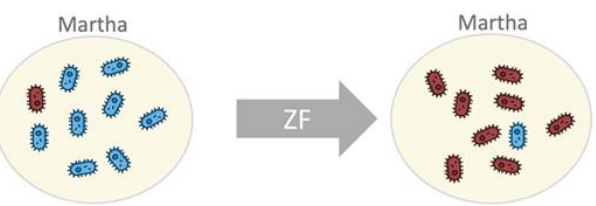

B) Population Level Selection of AMR

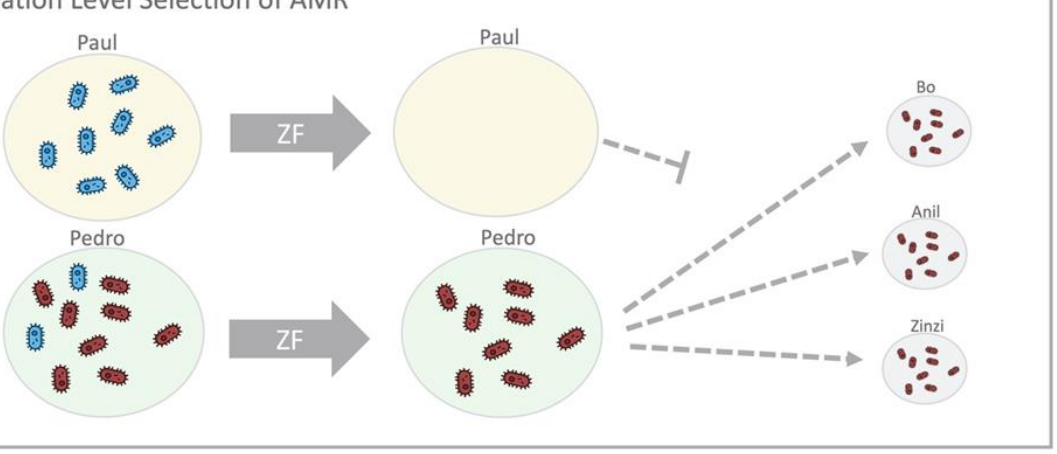

Figure 4. An illustration of individual- and population-level mechanisms that select for antimicrobial resistance (AMR) in commensal Neisseria, using the example of zoliflodacin (ZF). (A) In the individual level selection scenario (top), Martha takes ZF, and this eradicates the ZF-susceptible Neisseria (blue bacteria), leaving Martha with predominantly ZF-resistant Neisseria (red bacteria) post-treatment. (B) Recent studies have found that commensal Neisseria can be transmitted by kissing. Population-level selection of AMR by widespread use of ZF (bottom) works by eradicating the susceptible Neisseria from Paul and Pedro, leaving only resistant Neisseria to be transmitted by both of them to others.

If this line of reasoning is correct, then it would follow that high antimicrobial consumption would be manifested in earlier and higher AMR in commensal Neisseria than 
NG. Furthermore, there should be a correlation between antimicrobial consumption and antimicrobial susceptibility in commensal Neisseria. Whilst more evidence is required in this regard, the available evidence is generally supportive. The commensal Neisseria MICs of azithromycin and ceftriaxone are significantly higher in HIV preexposure prophylaxis (PrEP) clients in Belgium than those from the general Belgian population, whose consumption of these antimicrobials is considerably lower (unpublished data). In men who have sex with men (MSM) taking PrEP in Belgium, we have found that the median MIC for the most prevalent commensal, $N$. subflava, for example, increased from 1 in historical samples (1980-2000) to $176 \mathrm{mg} / \mathrm{L}$ in 2019 [15]. When we explored the potential drivers of this increase in resistance, we found that the azithromycin used for dual therapy of NG resulted in a macrolide consumption of 9.5 to 12 (DID) defined daily doses/1000 inhabitants/day $[33,34]$. This is approximately 4 to 7 times higher than the thresholds estimated to induce macrolide resistance in a range of bacterial species [35]. In vitro experiments demonstrated that NG was able to acquire macrolide resistance from DNA extracts from these highly macrolide-resistant $N$. subflavas via transformation [36]. The results for ceftriaxone were similar-there has been a large rightward shift in the MICs of commensal Neisseria in MSM taking PrEP and exposed to high levels of cephalosporins. These commensals were found to contain a number of mutations in pen $A$, por $B$, and $m \operatorname{tr} C D E$ that can be taken up by NG and result in macrolide and cephalosporin resistance [29].

There is also considerable supportive evidence for the resilience of commensal Neisseria. As already noted, one study found that oropharyngeal commensal Neisseria were as abundant 14 days post-therapy with ceftriaxone/azithromycin as pre-treatment [29]. This study also found that the median azithromycin and ceftriaxone MICs of all isolated Neisseria were higher in the post-treatment isolates $[15,29]$. Likewise, a study of commensal Neisseria in MSM in Vietnam found high cephalosporin MICs that were strongly correlated with recent receipt of a cephalosporin [14]. These findings suggest that whilst dual therapy is highly efficacious in eradicating NG and other susceptible Neisseria, it selects for macrolide- and cephalosporin-resistant commensal Neisseria (and other bacteria). In the case of oral streptococci, macrolide consumption has been shown to result in an increase in the proportion of isolates with macrolide resistance-an effect that persists for over 6 months [37]. Azithromycin's effect on the abundance of resistance-associated genes in the gastrointestinal tract may persist for four years $[38,39]$.

\section{Differences Stemming from Monospecies and Pan-Neisseria Approaches}

These findings suggest important difference between the monospecies and panNeisseria approaches as regarding therapeutic choices for NG (Figure 1; Table 1). The monospecies approach only considers the risk of resistance induction in NG. It is thus more likely to favour dual therapy, as this combination may minimise the risk of treatment failure, which could result in resistance [6]. The pan-Neisseria approach, however, incorporates the effect of proposed therapies on AMR in commensal Neisseria. It is likely to be more circumspect about dual therapy that includes azithromycin, especially considering that its long half-life means the drug is present for at least 2 to 4 weeks post-treatment in decreasing concentrations [40]. This azithromycin tail poses little risk of induction of AMR from a monospecies perspective (as the NG has been eradicated) [40] but a large risk for inducing AMR from a pan-Neisseria perspective, as commensal Neisseria are in the process of reconstituting their populations during this period $[9,30]$.

As already noted, dual therapy in conjunction with screening for NG in MSM PrEP cohorts results in very high consumption levels of macrolides-9.5 to 12 DID [33,34]. Whilst we do not know what the thresholds for the emergence of AMR are in commensal Neisseria, the fact that this consumption is 4- to 7-fold higher than the thresholds for inducing AMR in other organisms and the very high azithromycin MICs of commensal Neisseria spp. isolated from these PrEP cohorts suggests that the macrolide resistance threshold is considerably lower than 12 DID. 
To ascertain variables such as these resistance inducing thresholds, the pan-Neisseria approach would advocate introducing surveillance of commensal Neisseria antimicrobial susceptibility in key populations (Table 1). The other variables that could be obtained from such a process and be used to model the relationship between antimicrobial consumption and AMR in NG and commensals are detailed in Figure 2. This figure describes the six main pathways linking antimicrobial consumption and AMR in NG/commensal Neisseria. The relative importance of these pathways may vary by type of antimicrobial considered. Thus, macrolides such as azithromycin appear to be particularly adept at inducing NG resistance indirectly via the commensal pathway [18]. This has important implications for the use of macrolides for a range of infections/indications. Macrolides are frequently used for treatment of infections caused by organisms such as Chlamydia trachomatis, Mycoplasma genitalium and Streptococcus pneumoniae, where alternative agents are available [41-43]. They are also used long-term to prevent exacerbations of chronic obstructive pulmonary disease. The pan-Neisseria approach would recommend that bystander selection be considered when developing treatment guidelines for these infections.

An important criticism of the pan-Neisseria approach is that whilst HGT was important in the genesis of macrolide and cephalosporin resistance, these events were infrequent, and the majority of spread of this resistance has been clonal $[1,18,19]$. Whilst there is merit in this argument, there is also evidence that episodes of HGT in penA, $\operatorname{trCDE}, g y r A$ and other loci have been frequent in the pathogenic Neisseria $[16,17,20,21]$. Whilst there are no longitudinal studies evaluating the incidence of HGT in NG, a study that followed up with a cohort for 6 months found high incidence of HGT between N. meningitidis and N. lactamica. Evidence of HGT between these two Neisseria species during the followup period was detected in 15 loci in the two individuals that were co-colonised by both bacteria at baseline [44]. Finally, the clonal spread of these mosaic genes within NG is itself strongly influenced by antimicrobial consumption $[45,46]$. By including consideration of AMR in commensal Neisseria, one may be able to detect the emergence of AMR at an earlier and reversible stage and use this information to reduce consumption of the relevant antimicrobial in the target population.

\section{Application of the Pan-Neisseria Approach to Novel Treatments}

We end by illustrating how the pan-Neisseria approach could be used to evaluate the promising novel anti-NG agent, zoliflodacin, currently in phase 3 trials (Figure 2) [47]. Following the monospecies approach, current in vitro evaluations of this agent have been limited to assessing how easily zoliflodacin resistance can be induced in NG [48-50]. Likewise, assessments of the prevalence of pre-existing, resistance-conferring gyr $B$ mutations have been considered in NG and not commensal Neisseria [47]. A pan-Neisseria approach would complement these activities by including a panel of commensal Neisseria in these evaluations. If zoliflodacin resistance could be induced in commensal Neisseria relatively easily in vitro, then one could assess how easily this resistance could be transformed into NG in subsequent experiments.

It may be objected that we have no evidence of HGT ever having taken place in $g y r B$ in N. gonorrhoeae. Whilst this is true, we have good evidence that HGT in gyrA played an important role in the genesis of fluoroquinolone resistance in $N$. meningitidis. A study in Shanghai found that $99.3 \%$ of commensal Neisseria and $67.7 \%$ of $N$. meningitidis isolates were resistant to fluoroquinolones, and that HGT from commensals was responsible for fluoroquinolone resistance in over half the N. meningitidis isolates [16]. The most plausible reason for this extremely high prevalence of fluoroquinolone resistance in Neisseria species is the high consumption levels of fluoroquinolones in the general population [16]. In silico analyses of gonococcal and commensal gyr $A$ from around the world have revealed that HGT has played a similarly important role in the genesis of fluoroquinolone resistance in N. gonorrhoeae (unpublished data).

These findings provide the motivation to include surveillance of commensal Neisseria susceptibility to zoliflodacin in clinical trials and implementation projects of zoliflodacin. 
This could complement conventional AMR surveillance that monitors zoliflodacin MICs in NG. It would be particularly important to do in core groups with high exposure to anti-gonococcal therapies, such as PrEP cohorts [51]. Such a surveillance system could act as an early warning system for the emergence of zoliflodacin AMR in NG. One might find that zoliflodacin resistance was easily transformed from commensals into NG, and zoliflodacin AMR emerged faster in commensals than NG. If this was the case, then one could decide to switch/cycle the recommended NG treatment from zoliflodacin to another agent once predefined resistance thresholds in commensal Neisseria were crossed.

The pan-Neisseria approach builds on the insights of authors such as Bacquero et al., who have noted the utility of conceptualising epidemics of AMR as occurring simultaneously in multiple species [52]. They noted that epidemics of extended spectrum beta lactamase (ESBL) resistance in Gram negative bacteria occurred in a range of species rather than a single species. Excess use of cephalosporins resulted in outbreaks of ESBL producers in multiple species, partly driven by these species sharing the resistance-conferring enzymes with one another. In a similar vein, other authors have found correlations between fluoroquinolone resistance in various Gram negatives and N. gonorrhoeae at a country level [53]. In all cases, a key underlying driver of AMR is excessive antimicrobial consumption. This creates the rationale to select early warning species that can be used in surveillance programs to provide an alert when antimicrobial consumption is becoming excessive. Commensal Neisseria may be useful for this purpose, particularly in populations with a high STI incidence [14,15].

The pan-Neisseria approach raises a large number of important research questions. How does AMR in commensals vary by time and population (including core groups with different intensities of antimicrobial exposure)? Are there thresholds for the emergence of AMR in commensals? Is AMR reversible? How effective would antimicrobial cycling be to prevent AMR? What is the probability of HGT between Neisseria spp. in vivo? Do antimicrobial induced changes to commensal Neisseria populations have other adverse health effects?

Ultimately, all knowledge is underpinned by theory. An optimal theory of the determinants of gonococcal AMR should provide an accurate portrayal of all the important determinants in a way that illustrates the interrelationships and the relative importance of the various determinants and facilitates proportionate and effective responses [54]. If the theory conceals certain determinants, then it should be replaced by a theory which does not do this [54,55]. From the evidence we have reviewed, we conclude that a pan-Neisseria theoretical approach should be preferred to a monospecies approach, as it provides a more complete understanding of the genesis and spread of AMR in NG.

\section{Conclusions}

The development of new gonorrhoea treatment guidelines typically considers the resistance-inducing effect of the treatment only on Neisseria gonorrhoeae. Antimicrobial resistance in N. gonorrhoeae has, however, frequently first emerged in commensal Neisseria species and then been passed on to N. gonorrhoeae via transfer of the relevant resistance genes. This creates the rationale for considering the effect of gonococcal therapies on resistance in commensal Neisseria as well as on N. gonorrhoeae.

Author Contributions: C.K. wrote the first draft, all authors contributed to and read the final draft. All authors have read and agreed to the published version of the manuscript.

Funding: No funding was received for the work described in this manuscript.

Data Availability Statement: Not applicable.

Conflicts of Interest: The authors state that they have no conflict of interest. 


$\begin{array}{ll}\text { Abbreviations } \\ \text { AMR } & \text { antimicrobial resistance } \\ \text { AZM } & \text { azithromycin } \\ \text { CRO } & \text { ceftriaxone } \\ \text { GASP } & \text { Global Gonococcal Antimicrobial Surveillance Programme } \\ \text { NG } & \text { N. gonorrhoeae } \\ \text { ZF } & \text { zoliflodacin }\end{array}$

\section{References}

1. Harris, S.R.; Cole, M.J.; Spiteri, G.; Sánchez-Busó, L.; Golparian, D.; Jacobsson, S.; Goater, R.; Abudahab, K.; Yeats, C.A.; Bercot, B.; et al. Public health surveillance of multidrug-resistant clones of Neisseria gonorrhoeae in Europe: A genomic survey. Lancet Infect. Dis. 2018, 18, 758-768. [CrossRef]

2. Kenyon, C.; Buyze, J.; Wi, T. Antimicrobial Consumption and Susceptibility of Neisseria gonorrhoeae: A Global Ecological Analysis. Front. Med. 2018, 5. [CrossRef] [PubMed]

3. Lahra, M.M.; Enriquez, R. Australian Gonococcal Surveillance Programme Annual Report, 2016. Commun. Dis. Intell. 2018, 42. [CrossRef]

4. Yin, Y.-P.; Han, Y.; Dai, X.-Q.; Zheng, H.-P.; Chen, S.-C.; Zhu, B.-Y.; Yong, G.; Zhong, N.; Hu, L.-H.; Cao, W.-L.; et al. Susceptibility of Neisseria gonorrhoeae to azithromycin and ceftriaxone in China: A retrospective study of national surveillance data from 2013 to 2016. PLoS Med. 2018, 15, e1002499. [CrossRef]

5. Wi, T.; Lahra, M.M.; Ndowa, F.; Bala, M.; Dillon, J.-A.R.; Ramon-Pardo, P.; Eremin, S.R.; Bolan, G.; Unemo, M. Antimicrobial resistance in Neisseria gonorrhoeae: Global surveillance and a call for international collaborative action. PLoS Med. 2017, 14, e1002344. [CrossRef] [PubMed]

6. Unemo, M.; Ross, J.; Serwin, A.; Gomberg, M.; Cusini, M.; Jensen, J. Background review for the '2020 European guideline for the diagnosis and treatment of gonorrhoea in adults'. Int. J. STD AIDS 2021, 32, 108-126. [CrossRef]

7. Carpenter, C.M.; Bahn, J.M.; Ackerman, H.; Stokinger, H.E. Adaptability of Gonocoecus to Four Bacteriostatic Agents, Sodium Sulfathiazole, Rivanol Lactate, Promin, and Penicillin. Exp. Biol. Med. 1945, 60, 168-171. [CrossRef]

8. St. Cyr, S.; Barbee, L.; Workowski, K.A.; Bachmann, L.H.; Pham, C.; Schlanger, K.; Torrone, E.; Weinstock, H.; Kersh, E.N.; Thorpe, P. Update to CDC's Treatment Guidelines for Gonococcal Infection, 2020. MMWR. Morb. Mortal. Wkly. Rep. 2020, 69, 1911-1916.

9. Kenyon, C.R.; Schwartz, I.S. Effects of Sexual Network Connectivity and Antimicrobial Drug Use on Antimicrobial Resistance inNeisseria gonorrhoeae. Emerg. Infect. Dis. 2018, 24, 1195-1203. [CrossRef] [PubMed]

10. Dye, C.; Williams, B.G. The Population Dynamics and Control of Tuberculosis. Science 2010, 328, 856-861. [CrossRef]

11. Johnson, R.; Streicher, E.M.; Louw, G.E.; Warren, R.M.; van Helden, P.D.; Victor, T.C. Drug resistance in Mycobacterium tuberculosis. Curr. Issues Mol. Biol. 2006, 8, 97-111. [PubMed]

12. Worthington, R.J.; Melander, C. Combination approaches to combat multidrug-resistant bacteria. Trends Biotechnol. 2013, 31, 177-184. [CrossRef] [PubMed]

13. Lipsitch, M.; Samore, M.H. Antimicrobial Use and Antimicrobial Resistance: A Population Perspective. Emerg. Infect. Dis. 2002, 8, 347-354. [CrossRef] [PubMed]

14. Dong, H.V.; Pham, L.Q.; Nguyen, H.T.; Nguyen, M.X.B.; Nguyen, T.V.; May, F.; Le, G.M.; Klausner, J.D. Decreased Cephalosporin Susceptibility of Oropharyngeal Neisseria Species in Antibiotic-using Men Who Have Sex With Men in Hanoi, Vietnam. Clin. Infect. Dis. 2020, 70, 1169-1175. [CrossRef]

15. Laumen, J.G.E.; Van Dijck, C.; Abdellati, S.; Manoharan-Basil, S.S.; De Baetselier, I.; Martiny, D.; Crucitti, T.; Kenyon, C. Markedly Reduced Azithromycin and Ceftriaxone Susceptibility in Commensal Neisseria Species in Clinical Samples From Belgian Men Who Have Sex With Men. Clin. Infect. Dis. 2021, 72, 363-364. [CrossRef]

16. Chen, M.; Zhang, C.; Zhang, X.; Chen, M. Meningococcal Quinolone Resistance Originated from Several Commensal Neisseria Species. Antimicrob. Agents Chemother. 2019, 64. [CrossRef]

17. Ito, M.; Deguchi, T.; Mizutani, K.-S.; Yasuda, M.; Yokoi, S.; Ito, S.-I.; Takahashi, Y.; Ishihara, S.; Kawamura, Y.; Ezaki, T. Emergence and Spread of Neisseria gonorrhoeae Clinical Isolates Harboring Mosaic-Like Structure of Penicillin-Binding Protein 2 in Central Japan. Antimicrob. Agents Chemother. 2005, 49, 137-143. [CrossRef] [PubMed]

18. Wadsworth, C.B.; Arnold, B.J.; Sater, M.R.A.; Grad, Y.H. Azithromycin Resistance through Interspecific Acquisition of an EpistasisDependent Efflux Pump Component and Transcriptional Regulator inNeisseria gonorrhoeae. mBio 2018, 9. [CrossRef] [PubMed]

19. Banhart, S.; Selb, R.; Oehlmann, S.; Bender, J.; Buder, S.; Jansen, K.; Heuer, D. The mosaic mtr locus as major genetic determinant of azithromycin resistance of Neisseria gonorrhoeae, Germany, 2018. J. Infect. Dis. 2021. [CrossRef] [PubMed]

20. Yahara, K.; Nakayama, S.-I.; Shimuta, K.; Lee, K.-I.; Morita, M.; Kawahata, T.; Kuroki, T.; Watanabe, Y.; Ohya, H.; Yasuda, M.; et al. Genomic surveillance of Neisseria gonorrhoeae to investigate the distribution and evolution of antimicrobial-resistance determinants and lineages. Microb. Genom. 2018, 4, e000205. [CrossRef]

21. Mechergui, A.; Achour, W.; Ben Hassen, A. Antibiotic resistance among commensal Neisseria species. Rev. Med Microbiol. 2014, 25, 93-99. [CrossRef] 
22. Furuya, R.; Tanaka, M.; Onoye, Y.; Kanayama, A.; Saika, T.; Iyoda, T.; Tatewaki, M.; Matsuzaki, K.; Kobayashi, I. Antimicrobial resistance in clinical isolates of Neisseria subflava from the oral cavities of a Japanese population. J. Infect. Chemother. 2007, 13, 302-304. [CrossRef]

23. Sato-Suzuki, Y.; Washio, J.; Wicaksono, D.P.; Sato, T.; Fukumoto, S.; Takahashi, N. Nitrite-producing oral microbiome in adults and children. Sci. Rep. 2020, 10, 1-12. [CrossRef]

24. Aas, J.A.; Paster, B.J.; Stokes, L.N.; Olsen, I.; Dewhirst, F.E. Defining the Normal Bacterial Flora of the Oral Cavity. J. Clin. Microbiol. 2005, 43, 5721-5732. [CrossRef] [PubMed]

25. Willis, J.R.; Gabaldón, T. The Human Oral Microbiome in Health and Disease: From Sequences to Ecosystems. Microorganisms 2020, 8, 308. [CrossRef]

26. Yolken, R.; Prandovszky, E.; Severance, E.G.; Hatfield, G.; Dickerson, F. The oropharyngeal microbiome is altered in individuals with schizophrenia and mania. Schizophr. Res. 2020. [CrossRef] [PubMed]

27. Barbadoro, P.; Ponzio, E.; Coccia, E.; Prospero, E.; Santarelli, A.; Rappelli, G.G.; D’Errico, M.M. Association between hypertension, oral microbiome and salivary nitric oxide: A case-control study. Nitric Oxide 2021, 106, 66-71. [CrossRef] [PubMed]

28. Kraal, L.; Abubucker, S.; Kota, K.; Fischbach, M.A.; Mitreva, M. The Prevalence of Species and Strains in the Human Microbiome: A Resource for Experimental Efforts. PLoS ONE 2014, 9, e97279. [CrossRef]

29. de Block, T.; Laumen, J.G.E.; Van Dijck, C.; Abdellati, S.; De Baetselier, I.; Manoharan-Basil, S.S.; Van den Bossche, D.; Kenyon, C. Wgs of commensal neisseria reveals acquisition of a new ribosomal protection protein (Msrd) as a possible explanation for high level azithromycin resistance in Belgium. Pathogens 2021, 10, 384. [CrossRef]

30. Tedijanto, C.; Olesen, S.W.; Grad, Y.H.; Lipsitch, M. Estimating the proportion of bystander selection for antibiotic resistance among potentially pathogenic bacterial flora. Proc. Natl. Acad. Sci. USA 2018, 115, E11988-E11995. [CrossRef]

31. Van Dijck, C.; Laumen, J.G.E.; Manoharan-Basil, S.S.; Kenyon, C. Commensal Neisseria Are Shared between Sexual Partners: Implications for Gonococcal and Meningococcal Antimicrobial Resistance. Pathogens 2020, 9, 228. [CrossRef] [PubMed]

32. Kort, R.; Caspers, M.; Van De Graaf, A.; Van Egmond, W.; Keijser, B.; Roeselers, G. Shaping the oral microbiota through intimate kissing. Microbiome 2014, 2, 41. [CrossRef]

33. Kenyon, C.; De Baetselier, I.; Wouters, K. Screening for STIs in PrEP cohorts results in high levels of antimicrobial consumption. Int. J. STD AIDS 2020, 31, 1215-1218. [CrossRef]

34. Kenyon, C. We need to consider collateral damage to resistomes when we decide how frequently to screen for chlamydia/gonorrhoea in PrEP cohorts. AIDS 2019, 33, 155-157. [CrossRef] [PubMed]

35. Kenyon, C.; Manoharan-Basil, S.S.; Van Dijck, C. Is There a Resistance Threshold for Macrolide Consumption? Positive Evidence from an Ecological Analysis of Resistance Data from Streptococcus pneumoniae, Treponema pallidum, and Mycoplasma genitalium. Microb. Drug Resist. 2021. [CrossRef] [PubMed]

36. Abdelatti, S.; Gonzales, N.; De Block, T.; Manoharan-Basil, S.; Laumen, J.; De Baetselier, I.; Kenyon, C. Intraspecies transformation of msrD gene conferring high-level macrolide resistance in Neisseria subflava. In Proceedings of the ECCMID, 9-12 July 2021.

37. Malhotra-Kumar, S.; Lammens, C.; Coenen, S.; Van Herck, K.; Goossens, H. Effect of azithromycin and clarithromycin therapy on pharyngeal carriage of macrolide-resistant streptococci in healthy volunteers: A randomised, double-blind, placebo-controlled study. Lancet 2007, 369, 482-490. [CrossRef]

38. Doan, T.; Worden, L.; Hinterwirth, A.; Arzika, A.M.; Maliki, R.; Abdou, A.; Zhong, L.; Chen, C.; Cook, C.; LeBas, E.; et al. Macrolide and Nonmacrolide Resistance with Mass Azithromycin Distribution. N. Engl. J. Med. 2020, 383, 1941-1950. [CrossRef]

39. Jakobsson, H.E.; Jernberg, C.; Andersson, A.F.; Sjölund-Karlsson, M.; Jansson, J.K.; Engstrand, L. Short-Term Antibiotic Treatment Has Differing Long-Term Impacts on the Human Throat and Gut Microbiome. PLoS ONE 2010, 5, e9836. [CrossRef]

40. Kong, F.Y.S.; Horner, P.; Unemo, M.; Hocking, J.S. Pharmacokinetic considerations regarding the treatment of bacterial sexually transmitted infections with azithromycin: A review. J. Antimicrob. Chemother. 2019, 74, 1157-1166. [CrossRef]

41. Lanjouw, E.; Ouburg, S.; De Vries, H.; Stary, A.; Radcliffe, K.; Unemo, M. 2015 European guideline on the management of Chlamydia trachomatis infections. Int. J. STD AIDS 2015, 27, 333-348. [CrossRef]

42. Woodhead, M. Community-acquired pneumonia guidelines-An international comparison: A view from Europe. Chest 1998, 113, 183S-187S. [CrossRef]

43. Jensen, J.; Cusini, M.; Gomberg, M.; Moi, H. 2016 European guideline on Mycoplasma genitalium infections. J. Eur. Acad. Dermatol. Venereol. 2016, 30, 1650-1656. [CrossRef] [PubMed]

44. Pandey, A.; Cleary, D.W.; Laver, J.R.; Gorringe, A.; Deasy, A.M.; Dale, A.P.; Morris, P.D.; Didelot, X.; Maiden, M.C.J.; Read, R.C. Microevolution of Neisseria lactamica during nasopharyngeal colonisation induced by controlled human infection. Nat. Commun. 2018, 9, 4753. [CrossRef]

45. Osnes, M.N.; van Dorp, L.; Brynildsrud, O.B.; Alfsnes, K.; Schneiders, T.; Templeton, K.E.; Yahara, K.; Balloux, F.; Caugant, D.A.; Eldholm, V. Antibiotic Treatment Regimes as a Driver of the Global Population Dynamics of a Major Gonorrhea Lineage. Mol. Biol. Evol. 2021, 38, 1249-1261. [CrossRef]

46. Kenyon, C.; Laumen, J.; Van Dijck, C.; De Baetselier, I.; Abdelatti, S.; Manoharan-Basil, S.S.; Unemo, M. Gonorrhoea treatment combined with population-level general cephalosporin and quinolone consumption may select for Neisseria gonorrhoeae antimicrobial resistance at the levels of NG-MAST genogroup: An ecological study in Europe. J. Glob. Antimicrob. Resist. 2020, 23, 377-384. [CrossRef] 
47. Unemo, M.; Ahlstrand, J.; Sánchez-Busó, L.; Day, M.; Aanensen, D.; Golparian, D.; Jacobsson, S.; Cole, M.J.; Torreblanca, R.A.; Ásmundsdóttir, L.R.; et al. High susceptibility to zoliflodacin and conserved target (GyrB) for zoliflodacin among 1209 consecutive clinical Neisseria gonorrhoeae isolates from 25 European countries, 2018. J. Antimicrob. Chemother. 2021, 76, 1221-1228. [CrossRef]

48. Foerster, S.; Drusano, G.; Golparian, D.; Neely, M.; Piddock, L.J.V.; Alirol, E.; Unemo, M. In vitro antimicrobial combination testing of and evolution of resistance to the first-in-class spiropyrimidinetrione zoliflodacin combined with six therapeutically relevant antimicrobials for Neisseria gonorrhoeae. J. Antimicrob. Chemother. 2019, 74, 3521-3529. [CrossRef] [PubMed]

49. Foerster, S.; Golparian, D.; Jacobsson, S.; Hathaway, L.J.; Low, N.; Shafer, W.M.; Althaus, C.L.; Unemo, M. Genetic Resistance Determinants, In Vitro Time-Kill Curve Analysis and Pharmacodynamic Functions for the Novel Topoisomerase II Inhibitor ETX0914 (AZD0914) in Neisseria gonorrhoeae. Front. Microbiol. 2015, 6, 1377. [CrossRef] [PubMed]

50. Alm, R.A.; Lahiri, S.D.; Kutschke, A.; Otterson, L.G.; McLaughlin, R.E.; Whiteaker, J.D.; Lewis, L.A.; Su, X.; Huband, M.D.; Gardner, H.; et al. Characterization of the Novel DNA Gyrase Inhibitor AZD0914: Low Resistance Potential and Lack of Cross-Resistance in Neisseria gonorrhoeae. Antimicrob. Agents Chemother. 2014, 59, 1478-1486. [CrossRef] [PubMed]

51. Lewis, D.A. The role of core groups in the emergence and dissemination of antimicrobial-resistantN gonorrhoeae. Sex. Transm. Infect. 2013, 89, iv47-iv51. [CrossRef]

52. Baquero, F.; Cocque, T.M.; Canton, R. Allodemics. Lancet Infect. Dis. 2002, 2, 591-592. [CrossRef]

53. Kenyon, C.; Manoharan-Basil, S.S.; Van Dijck, C. Gonococcal resistance can be viewed productively as part of a syndemic of antimicrobial resistance: An ecological analysis of 30 European countries. Antimicrob. Resist. Infect. Control. 2020, 9, 1-9. [CrossRef] [PubMed]

54. Krieger, N. Epidemiology and the web of causation: Has anyone seen the spider? Soc. Sci. Med. 1994, 39, 887-903. [CrossRef]

55. Susser, M.; Susser, E. Choosing a future for epidemiology: II. From black box to Chinese boxes and eco-epidemiology. Am. J. Public Health 1996, 86, 674-677. [CrossRef] [PubMed] 